\title{
Status of Resistive Magnets in the LHC Injectors Chain
}

\author{
D. Tommasini, J. Bauche, D. Bodart, R. Lopez, A. Newborough, S. Sgobba, P. Thonet, and T. Zickler
}

\begin{abstract}
About 4650 normal conducting magnets are presently installed in the CERN accelerators complex, more than 3000 of them belonging to the LHC injector chain and 163 installed in the LHC. The oldest magnets have been in operation for 50 years, and some of them are submitted to aggressive conditions, either in terms of radiation, extreme water cooling conditions or temperature. The smallest magnets in the linacs weigh a few kilograms, whilst each of the main magnets of the Proton Synchrotron weighs 33 tons. The paper reviews the status of these magnets and gives some examples of findings and relevant recent actions undertaken to ensure their reliable operation in the coming years.
\end{abstract}

Index Terms-Accelerator magnets, resistive magnets.

\section{INTRODUCTION}

$\mathbf{T}$ HE particle beams colliding in the Large Hadron Collider (LHC) are provided by a complex of injectors (Fig. 1) constituted of about 3200 conventional magnets, without counting the fast injection/extraction magnets.

Protons, obtained by removing electrons from hydrogen atoms, are injected from the linear accelerator LINAC2 into the Proton Synchrotron (PS) Booster, then the PS, followed by the Super Proton Synchrotron (SPS) and, after traveling through either the TI2 Transfer Line (clockwise beam) or the TI8 Transfer Line (anticlockwise beam) to the LHC.

Lead ions, obtained from a source of vaporized lead, are injected from the linear accelerator LINAC3 and, instead of passing through the PS Booster, pass through the Low Energy Ion Ring (LEIR) before reaching the PS. They then follow the same route to the LHC as the protons.

Though identified as "conventional" in opposition to "superconducting magnets", the also called "normal conducting" or "resistive" magnets used in these accelerators are in several cases relatively "non-conventional", as for example the "four-in-one" magnets of the Proton Synchrotron Booster.

About 250 different types of magnets are installed in the LHC injectors chain. The lightest magnets, typically in the linacs, are just a few kilograms; the heaviest are the combined function main units of the PS, weighing about 33 tons, which are also among the oldest operating since 1959.

Manuscript received October 19, 2009.First published February 17, 2010; current version published May 28, 2010.

The authors are with CERN, 1211 Geneva 23, Switzerland (e-mail: davide. tommasini@cern.ch).

Color versions of one or more of the figures in this paper are available online at http://ieeexplore.ieee.org.

Digital Object Identifier 10.1109/TASC.2009.2039203

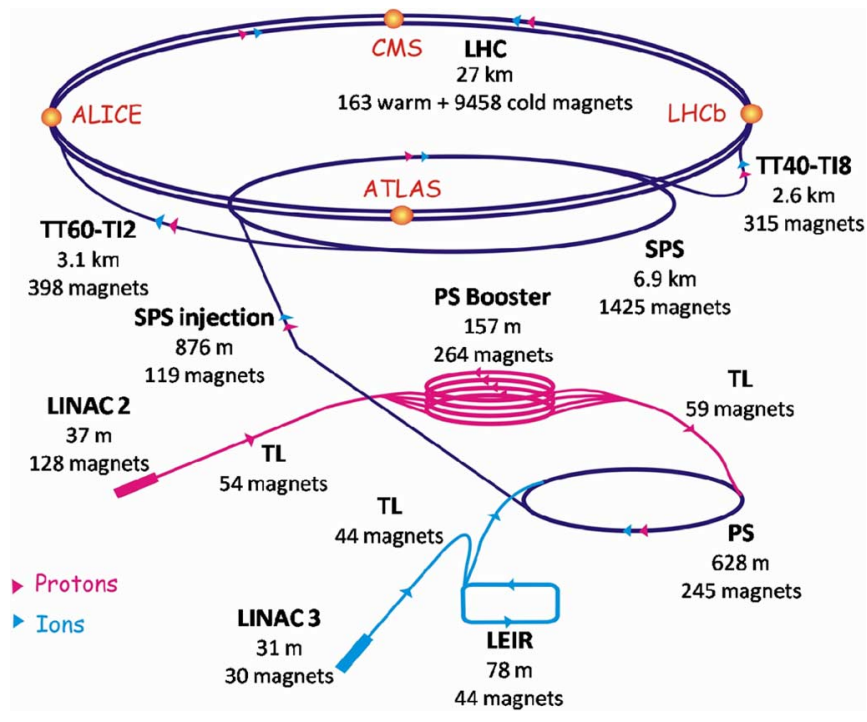

Fig. 1. The LHC injectors complex.

A failure, even temporarily, of almost any of the magnets belonging to the injector chain would stop the operation of all accelerators downstream in the chain.

To ensure the highest standards of reliability, reactive and preventive maintenance strategies are well established as part of the machine operation activities.

These actions allow operation of the magnets in the LHC injector complex with a low number and duration of failure events.

\section{Reactive Maintenance And Spare Policy}

During periods of accelerator operation a 24 hour 7 days a week standby service ensures an immediate reaction to any magnet fault. The specific strategy of intervention, either a temporary or definitive fix or even a magnet replacement with a spare unit, depends on many factors such as the type of fault, its localization, the radiation dose and the possible proximity to a programmed machine stop. In the case when a magnet is replaced by a spare, the defective unit is, when possible, repaired to become a new spare magnet.

\section{A. Spare Policy}

With few exceptions, each magnet type has spare coils, and most types have entire spare magnets. The number and type of spares depends on the probability of a failure, on the number of installed magnets and on the relevant induced risk of a magnet failure on the accelerator chain.

For example having spare coils and no spare magnets can be accepted where their replacement takes a short time or in 
secondary lines not directly concerned in the injection to the LHC. On the other hand the large quantity of the SPS main dipoles, the time required to possibly replace defective coils, the recurrently needed regular maintenance imposes for these magnets to have a relatively large number of spare units.

Finally, there are specific locations, for example close to the injection/extraction regions, machine collimation and dump, where the radiation doses are too high to do an important intervention on a defective magnet such as replacing its coils; such cases need spare magnets.

There are about 600 spare magnets to be possibly used in the LHC injectors. In many cases these magnets were built several decades ago and a campaign of certification of their status is presently under way.

\section{Preventive Maintenance}

The preventive maintenance is aimed at preserving and possibly enhancing the magnets reliability during the operation of the accelerators. It is difficult to find the correct balance between the cost for implementing a program of preventive maintenance and its benefits.

The cost does not include only the one of personnel and materials, but also the time spent for required machine stops, the exposure of personnel to ionizing radiation, the infrastructure and the logistics servicing the operations of preventive maintenance.

On the other hand, though in certain cases the benefit is immediate and obvious, for example when a scheduled inspection identifies a defective magnet during a machine stop, in other cases the real usefulness of the action may not be immediately visible.

At CERN the preventive maintenance of resistive magnets is based on:

- analysis of failures;

- regular inspections and checks in the accelerators;

- programmed replacement of equipment;

- corrective actions and consolidation programs;

\section{A. Analysis of Failures}

The analysis of failures defines most of the preventive maintenance policy.

When a failure shows signs of a statistical recurrence, different approaches can be decided depending on its impact on the operation of the accelerators. At one extreme, the major recurrent failures which were affecting the PS Booster, the PS and the SPS main magnets around the year 2000 have been treated in important consolidation programs which will be discussed later. On the other hand unexpected failures affecting magnets built in single or few units may trigger the need of making available not only spare coils but one or more complete spare magnets.

Fig. 2 summarizes the quantity of failures observed in magnets of the CERN injectors, including the relevant Transfer Lines (TL) during the first semester of operation, since March until September 2009.

Most of these failures were detected during regular inspections in the occasion of programmed technical stops: this applies in particular for water leaks in the SPS. The machine stops

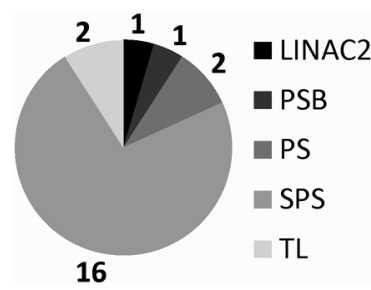

Fig. 2. Number of magnet failures in the LHC injectors, first semester 2009

due to magnet failures were 5 , producing 83 hours of machine down-time.

The main failure events were a short circuit of the magnet bus bars in the PS, needing about 24 hours for a temporary fix (the bus bars were thereafter replaced during a programmed technical stop) and a coil inter-turn short circuit in a SPS main dipole, producing 26 hours of machine stop to replace the defective magnet.

\section{B. Regular Inspections and Checks in the Accelerators}

Regular inspections and checks on magnets and on their ancillary systems, such as cooling circuits and interlocks, are performed during the accelerators technical stops and during the regular shut-down periods. Depending on the duration of the stop, these inspections and checks may include:

- visual checks with magnets not powered to detect evident problems like water leaks or mechanical damage;

- auditive checks while the magnets are pulsed, to reveal for example failures in the clamping of magnet coils;

- thermographic inspections of critical electrical joints, for example in the corrector circuits of the PS main magnets;

- dielectric tests and electrical continuity tests.

The frequency and duration of inspections in zones with high radiation doses are very limited and typically associated with interventions of reactive maintenance following a failure.

\section{Programmed Replacement of Equipment}

Possible replacements of magnets or of coils, in addition to the case of failures during the machine operation, are decided on the basis of the regular inspections and tests. In certain cases, as for the auxiliary magnets in the PS and most of all for the main magnets in the SPS, every year part of the magnets are replaced by reconditioned spare magnets.

In the SPS about 10 dipole and 5 quadrupole magnets are replaced every year: the units are chosen on the basis of the results of visual/auditive campaigns in the whole SPS.

\section{Consolidation Programs}

Consolidation programs are established in case of recurrent failures affecting the reliable operation of the accelerators. The following paragraphs describe the three main consolidation programs carried on magnets during the last decade.

\section{The PS Booster Magnets Consolidation}

Since 1998 the PS Booster operation had to be stopped frequently due to water leaks on the rubber flexible hoses and joints 


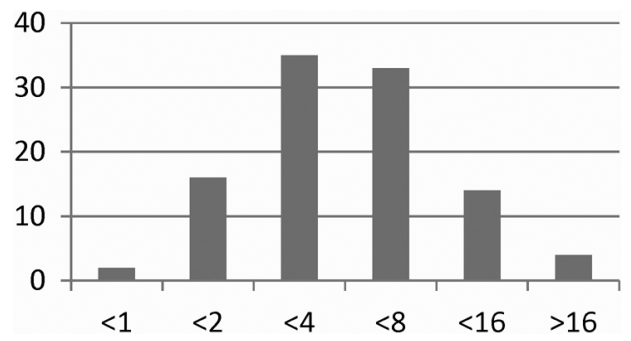

Fig. 3. Distribution of integrated radiation doses $[\mathrm{MGy}]$ in the PS magnets from 1959 to 2004, before the consolidation program.

used for the inter-coil hydraulic connections of the main magnets. The situation exploded at the machine start-up at the end of the 2005/2006 shutdown, when over 60 leaks were found on the cooling circuits. Two main types of failures were identified: the systematic degradation of the two-layers EDPM water hoses, with breakage of the reinforcement between the two layers due to aging (radiation, thermal and humidity), and the embrittlement of the seals leading to cracks.

During the shutdown 2006/2007 all 160 cooling circuits were modified to integrate new stainless steel manifolds.

\section{The PS Magnets CONSOLIDATION}

Following a regular inspection and tests performed on the main magnets during the 2002/2003 winter shutdown, two magnets failed to high voltage checks between coils and ground. The analysis of the failure and a detailed inspection of all other installed magnets, which were operating since 1959 , evidenced the heavy degradation of the insulation to ground of the main coils and of the cables used for the pole face correction windings (PFW). It was then decided to launch a major consolidation program to replace all main coils and PFW of the weakest 50 magnets, out of the 100 (plus one as reference magnet) installed in the PS.

The choice of the magnets to refurbish was based on the following criteria:

- the integrated radiation dose since 1959 (Fig. 3);

- the expected future levels of radiation;

- the state of the dielectric insulation of the main coils;

- the state of the PFW cables and internal connections;

- the voltage seen by the main coils during operation.

This program, detailed in [1], has been successfully completed during the 2008/2009 shutdown.

\section{THE SPS MAGNETS CONSOLIDATION}

The main recurrent failures affecting the SPS magnets are due to erosion of the copper water collectors of the cooling circuits and to the cyclic forces acting on the coils producing a progressive degradation of their clamping system. The first issue was particularly affecting the 254 dipoles produced by Lintott: six leaks were identified between 1986 and 2004, while in 2004, after 28 years of operation, seven events appeared in the same year. Leaks were generally due to erosion-corrosion (Fig. 4): the velocity of the coolant (demineralized water) in the circuits

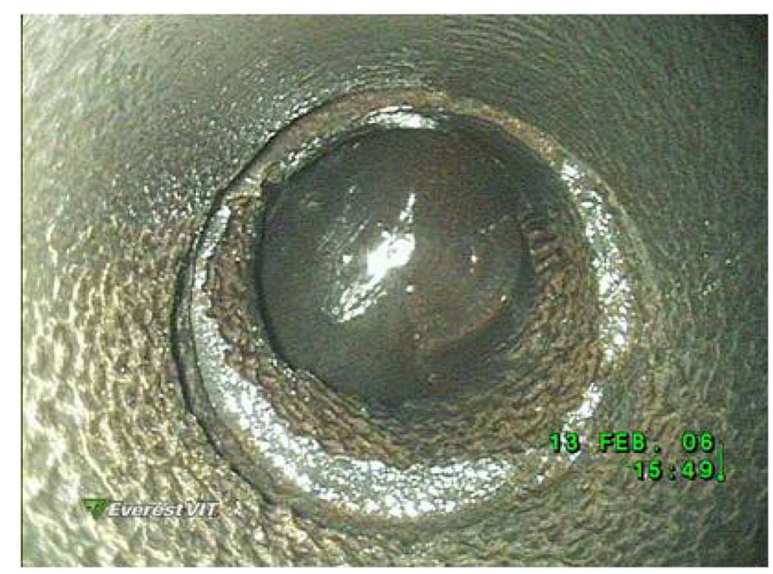

Fig. 4. Large patches of undercut pits characteristic of severe attack by erosioncorrosion of copper, observed by endoscopic inspection of the $13 \mathrm{~mm}$ diameter internal walls of the pipes of collectors of SPS dipole magnets.

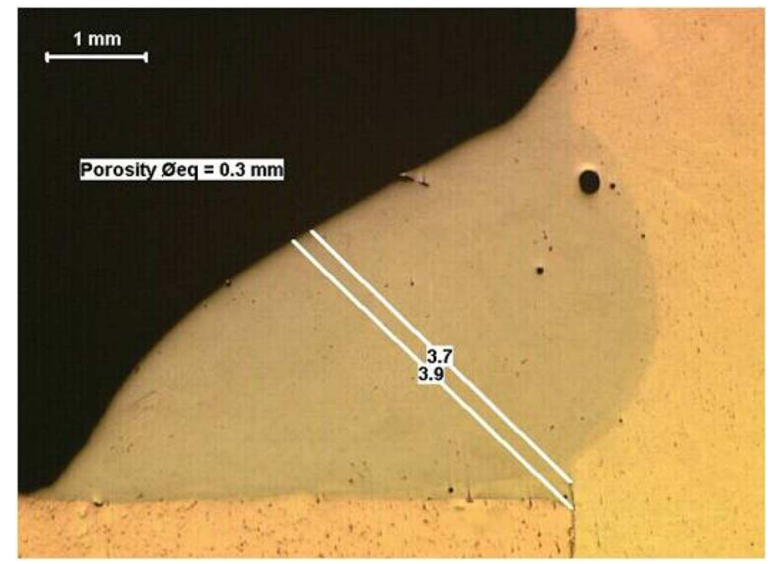

Fig. 5. Metallographic cross-cut of a fillet braze-weld. The minimum specified throat thickness is $2 \mathrm{~mm}$.

is locally as high as $9 \mathrm{~m} / \mathrm{s}$. For comparison, the maximum accepted water tubular design velocity for pure coppers are in the range of $1.5 \mathrm{~m} / \mathrm{s}$, since copper in pure waters exhibits a significantly velocity-dependent corrosion rate.

A systematic repair of the cooling circuit of 254 dipole magnets was performed during three campaigns coinciding with the shutdowns of 2006-07, 2007-08 and 2008-09. Repairs were carried out in a dedicated and specially prepared cavern of the SPS tunnel. The repair process deployed a complex organization of activities and allowed to treat about 85 magnets every shut-down [2].

The standard repair solution was based on braze-welding UNS C90700 fittings to OFE copper busbars through a TIG assisted process (Fig. 5). The filler metal is the self-fluxing alloy BCuP-3. This alloy avoids the application of activated brazing fluxes whose residues might be corrosive, particularly for nearby stainless steel vacuum components.

Compared to standard TIG welding or a conventional brazing process, braze-welding allows the heat loads to be minimized. A cooling device close to the weld joint permitted to limit even more the temperature rise during braze-welding. Temperature control is of paramount importance, since the nearby coil insulation would be damaged by overheating: at $50 \mathrm{~mm}$ from the 
weld axis the monitored temperature can only exceed $100{ }^{\circ} \mathrm{C}$ for a total time shorter than $2 \mathrm{~min}$, while a temperature of 120 ${ }^{\circ} \mathrm{C}$ shall never be exceeded.

In parallel to the repair campaigns, systematic corrosion studies based on electrochemical tests, and a large scale corrosion and erosion-corrosion test based on a circuit of demineralized water representative of the SPS magnet cooling system were performed.

Laboratory results and results of tests carried out on samples of different $\mathrm{Cu}$ alloys, braze-welded with different fillers and submitted to representative conditions of water flow, confirm the full adequacy of the adopted material choice and weld solutions [3].

Systematic destructive tests on weld witness sample, including tensile test of the joints, pressure tests and metallographic inspections, systematic temperature monitoring and recording during welding, pressure and electrical tests on the repaired magnets were carried out all during the three campaigns. The repaired units shall provide a fully reliable operation of the cooling circuits during the next decades.

\section{The TRANSFER LINES FROM SPS TO LHC}

The 348 main dipole magnets, 179 main quadrupoles and 93 corrector magnets for TI 2 and TI 8 transfer lines from the SPS to the LHC have been built by the Budker Institute for Nuclear Physics (BINP) in Novosibirsk [4], [5], as part of the contribution of the Russian Federation to the LHC project.

The injection tests into the LHC have shown that these magnets successfully behave as expected, with the exception of a sextupole component in the dipoles, observed by beam measurements, of about $4 \cdot 10^{-4}$ of the dipole component strength @ $25 \mathrm{~mm}$ from the beam axis, and recently confirmed by magnetic simulations of the magnet pole profile design [6]. The linear optics of the TI 2 and TI 8 lines can be trimmed to compensate this effect [7].

\section{ThE LHC RESISTIVE MAGNETS}

There are 155 resistive magnets in the LHC [8]. It is expected some of them, in particular the ones in the two cleaning regions LSS3 and LSS7, will reach radiation doses of several tens of MGy in less than 10 years. Possible future interventions on these magnets will have to be carefully planned and appropriate handling equipment is being developed to reduce the personnel exposition to radiations within acceptable limits.

\section{Future TRENDS}

The present complex of CERN injectors is not only used for the LHC, but also supplies beams to other experiments. To preserve its efficiency for several decades two directions are being pursued: further consolidation actions on the existing accelerators and the construction of new injectors.

\section{A. Consolidation of the Existing Accelerators}

It is planned to consolidate the number of spares of the most critical components, such as the PFW and the bus bars of the PS machines and other magnets in certain cases built as single units but essential for the operation of the injectors.

\section{B. Construction of New Injectors}

The strategy for a progressive constitution of a new injectors chain [10] is based on the need of replacing the oldest machines with new ones to preserve the reliability of the CERN accelerators complex for the next decades, possibly removing present limitations as for example the space charge effects in the SPS by increasing its injection energy to $50 \mathrm{GeV}$ by a new synchrotron replacing the PS.

The first step in this process is the construction of Linac4, presently under way [11], [12], a $160 \mathrm{MeV}$ proton linear accelerator which will replace in 2014 the present $50 \mathrm{MeV}$ Linac2.

\section{CONCLUSION}

After the completion in 2009 of two major consolidation programs on the PS and the SPS main magnets, the status of the CERN resistive magnets appears adequate for providing a reliable beam to the LHC in the next decade.

Beyond 2020 the PS will be more than 60 years old: either its complete renovation, possibly with a redesign of new main magnets, or the construction of a new accelerator, like PS2, has to be considered.

\section{REFERENCES}

[1] D. Bodart, W. Kalbreier, K.H. Meß, A. Newborough, and T. Zickler, "Consolidation of the 45-years old PS main magnet system," in EPAC 06, Edinburgh, Scotland.

[2] S. Sgobba, G. Favre, C. Podevin, and S. Prodon, Work-package for the repair of the cooling circuit of the SPS dipole magnets to be performed during the winter shutdown of 2006-07, 2007-08, 2008-09, CERNEDMS documents 783640, 861711, 944538.

[3] M. Flourez, Etude comparative du soudobrasage et de la résistance à la corrosion dans le cadre de la réparation des aimants dipolaires du SPS, Diploma work, IUT Bordeaux 1, CERN-EDMSdocument 850218, 2007.

[4] V. Anashin et al., "The bending magnets for the LHC injection transfer lines," IEEE Trans. Applied Superconductivity, vol. 12, no. 1, pp. 120-124, Mar. 2002.

[5] I. Chertok et al., "The quadrupole magnets for the LHC injection transfer lines," IEEE Trans. Applied Superconductivity, vol. 10, no. 1, pp. 154-157, Mar. 2000.

[6] J. Bauche, to be published.

[7] M. Meddahi, I. Agapov, K. Fuchsberger, B. Goddard, W. Herr, V. Kain, V. Mertens, and E. Gianfelice, "Machine studies during beam commissioning of the SPS-to-LHC transfer lines," in Presented at the Particle Accelerator Conference (PAC09), Vancouver, Canada, May 4-8, 2009.

[8] D. Gurov et al., "Normal-conducting separation and compensation dipoles for the LHC experimental insertions," IEEE Trans. Applied Superconductivity, vol. 16, no. 2, pp. 253-256, Jun. 2006.

[9] R. Garoby, "Upgrade issues for the Cern accelerator complex," in Proceedings of EPAC-08, Genova, Italy.

[10] R. Garoby, G. Bellodi, F. Gerigk, K. Hanke, A. Lombardi, M. Pasini, C. Rossi, E. Sargsyan, and M. Vretenar, "Linac4, a new injector for the CERN PS booster," in EPAC 06, Edinburgh, Scotland.

[11] F. Gerigk, C. Carli, R. Garoby, K. Hanke, A. M. Lombardi, R. Maccaferri, S. Maury, C. Rossi, and M. Vretenar, "Construction Status of Linac 4," in PAC-09, Vancouver, Canada.

[12] T. Zickler, "Status of the new Linac 4 magnets," in IEEE Trans. Appl. Supercond., Jun. 2010, vol. 20, no. 3. 